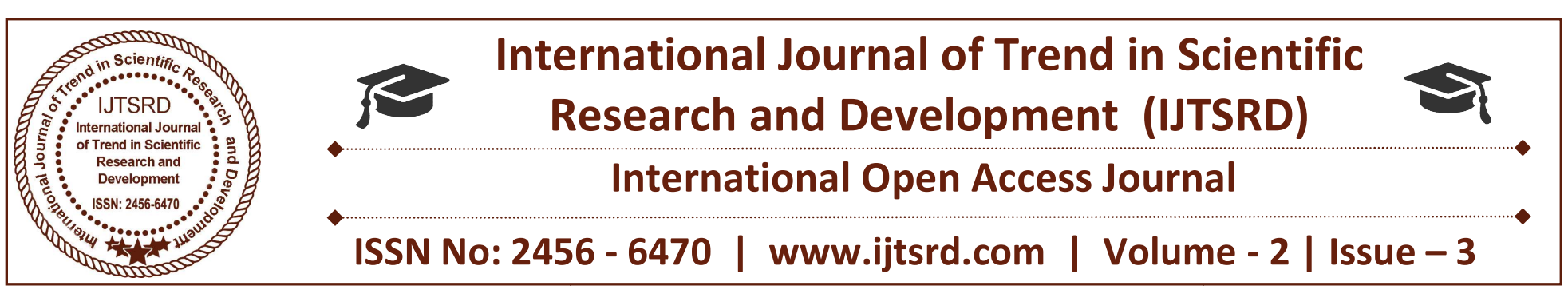

\title{
Review on CDSS implementation with CDA generation and integration for health information exchange in cloud
}

\author{
Pooja N. Umekar \\ Department of Computer Science \& \\ Engineering, \\ Dr. Rajendra Gode Institute of \\ Research and Technology, \\ Amravati, Maharashtra, India
}

\author{
Dr. H R. Deshmukh \\ HOD, Department of Computer \\ Science \& Engineering, Dr. \\ Rajendra Gode Institute of Research \\ and Technology, Amravati, \\ Maharashtra, India
}

\author{
Prof O A. Jaisinghani \\ Assistant Professor, Dr. Rajendra \\ Gode Institute of Research and \\ Technology, Amravati, \\ Maharashtra, India
}

\section{ABSTRACT}

Successful Deployment of EHR helps to improve patient safety and quality of care, but it has the prerequisite of interoperability between health information exchange at different hospital. The clinical document architecture developed HL7 is core document standard to ensure such interoperability and propagation of this document. Unfortunately, hospitals are reluctant to adopt interoperable HIS due to its deployment cost except for in a handful countries. A problem arises even when more hospital s start using the CDA document format because the data scattered in different documents are hard to manage. CDA document generation and integration Open API service based on cloud computing through which hospitals are enabled to conveniently generate CDA document per patient into a single CDA document and physician and patients can browse the clinical data in chronological order. CDA system of CDA document generation and integration is based on cloud computing and the service is offered in Open API.

A clinical decision support system (CDSS) is a health information technology system that is designed to provide physicians and other health professionals with clinical decision support (CDS), that is assistance with clinical decision -making tasks. A working definition has been proposed by Robert Hayward of the Centre for Health Evidence: "Clinical decision support systems link health observations with health knowledge to influence health choices by clinicians for improved health. Our system will implement CDSS clinical decision support system it the system looking towards the clinical decision support from existing CDA system provided information. CDSS will help the doctor's do the diagnosis of the patient from the existing CDA system. So that the time will be saved of the patient or if the patient is unable to bring or collect the health record the doctor's will be able to detect the particular disease.

Keywords: Health information exchange, HL7, CDA, cloud computing, software as a service, CDSS

\section{INTRODUCTION}

Electronic health record is the systematize collection of patient and population electronically stored information in digital format[w]. these record can be shared across different health care sitting. record are shared through network connected, enterprise vide information system or other information network and exchanges.EHR may include range of data including demographic medical history, medication and allergies, immunization status, laboratory test result, radiology images, vital signs, personal statestic like age and weight and billing information[w]. Health Level Seven has established CDA as a major standard for clinical documents. CDA is a document markup standard that specifies the structure and semantics of 'clinical documents' for the purpose of exchange. The 
first version of CDA was developed in 2001 and Release 2 came out in 2005 [1]. Many projects adopting CDA have been successfully completed in many countries. Active works are being done on improving semantic interoperability based on open EHR and CEN13606.

\section{Literature Review}

Health Level Seven has established CDA as a major standard for clinical documents [2]. CDA is a document markup standard that specifies the structure and semantics of 'clinical documents' for the purpose of exchange. The first version of CDA was developed in 2001 and Release 2 came out in 2005 [1]. Many projects adopting CDA have been successfully completed in many countries [3], [4], [5]. Active works are being done on improving semantic interoperability based on open EHR and CEN13606 [6], [7]. To establish confidence in HIE interoperability, more HIS's need to support CDA. However, the structure of CDA is very complex and the production of correct CDA document is hard to achieve without deep understanding of the CDA standard and sufficient experience with it. In addition, development the HIS platforms for hospitals vary so greatly that generation of CDA documents in each hospital invariably requires a separate CDA generation system. Also, hospitals are very reluctant to adopt a new system unless it is absolutely necessary for provision of care. As a result, the adoption rate of EHR is very low except for in a few handful countries such as New Zealand or Australia [8]. In the USA, the government implemented an incentive program called the Meaningful Use Program to promote EHR adoption among hospitals [9]. When a patient is diagnosed at a clinic, a CDA document recording the diagnosis is generated. The CDA document can be shared with other clinics if the patient agrees. The concept of family doctor does not exist in Korea, hence it is common for a patient to visit a number of different clinics.

The exchange of CDA document is triggered in the following cases: when a physician needs to study a patient's medical history; when referral and reply letters are drafted for a patient cared by multiple clinics; when a patient is in emergency and the medical history needs to be reviewed. It takes increasing amount of time for the medical personnel as the amount of exchanged CDA document increases because more documents means that data are distributed in different documents. This significantly delays the medical personnel in making decisions. Hence, when all of the CDA documents are integrated into a single document, the medical personnel is empowered to review the patient's clinical history conveniently in chronological order.

\section{PROPOSED OBJECTIVE :}

\subsection{CDA DOCUMENT :}

Data Items in CCD Header and Sections in the CCD Body

\section{CDA location $\quad$ Data items}

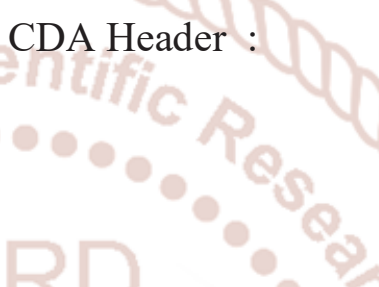

Document Information

(creation time, template ID, language code, purpose) Patient's information (ID, name, gender, birth date) Author's information (ID, name, represented organization) Organization's information (name, address, phone number)

CDA Body :

Payers Advance

Directives Support

Functional Status

Problems Family History

Social History

Allergies Medications

Medical Equipment

Vital Signs Results Procedures

Encounters Plan of Care

\subsection{CLOUD COMPUTING :}

This refers to both the applications delivered as service over internet and the hardware and systems software in the data centers that provide those services [10]

- Diagram of architecture of our CDA generation system based on cloud 
International Journal of Trend in Scientific Research and Development (IJTSRD) ISSN: 2456-6470

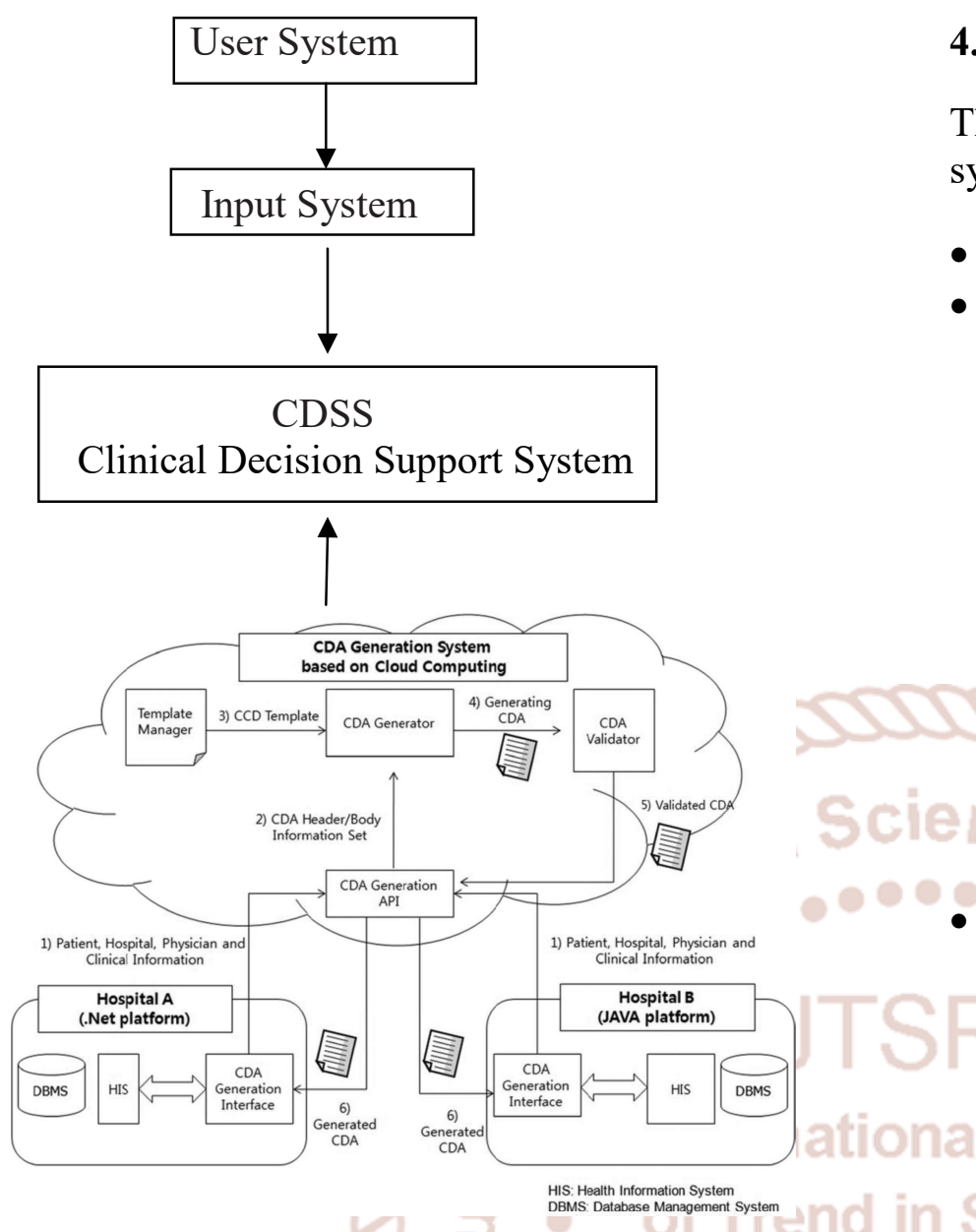

\subsection{CDA Generation System Based on Cloud Computing :}

The above diagram shows the overall architecture of how CDA documents can be generated on the health information systems of other hospitals by using our cloud computing based CDA generation systems .

1. CDA generation API generates CDA documents on cloud .

2. CDA generation Interface uses the API provided by the cloud and relays the input data and receives CDA documents generated on cloud .

3. Template manager is responsible for managing the CDA documents generated in cloud server.

4. CDA generator collects patients data from hospital and generates CDA documents in template .

5. CDA validator inspects whether the generated CDA documents complies with a CDA document complies with a CDA schema standards .

\section{Technical Specifications And Result Analysis:}

The technologies which are used to implement the system are:

- Java jdk.6.0

- Eclipse:In computer programming, Eclipse is an integrated development environment (IDE). It contains a base workspace and an extensible plugin system for customizing the environment. Written mostly in Java, Eclipse can be used to develop applications. By means of various plugins, Eclipse may also be used to develop applications in other programming languages: Ada, ABAP, C, C++, COBOL, Fortran, Haskell, JavaScript, Lasso, Lua, Natural, Perl, PHP, Prolog, Python, R, Ruby (including Ruby on Rails framework), Scala, Clojure, Groovy, Scheme, and Erlang.

- MySQL is open source relational database system. It is static. Database size is unlimited in MySQL. MySQL support Java. MySQL does not support except \& intersect operation. MySQL does not have resource limit. MySQL is available under GPL proprietary license. The MySQL development project has made its source code available under the term of the GNU General Public License, as well as under a variety of proprietary agreements. MySQL is a popular choice of database for used in web application. MySQL is written in $\mathrm{C}$ and $\mathrm{C}++$.

\section{Advantages:}

- Construction of a cloud computing environment and deployment of CDA generation and integration system based on it.

- Generation of CDA documents on different developer platforms through Cloud.

\section{Limitations:}

- As a number of HIE based on CDA documents increases, interoperability is achieved, but it also brings a problem while managing various CDA documents per patient becomes inconvienent as the clinical information for each patient is scattered in different documents .

- The followsing problems were encountered while developing our CDA document generation and integration system. First, the default language of the Amazon Cloud OS is US English and it did not adequately handle Korean language. 


\section{Conclusion:}

In this paper interoperability between hospitals not only helps improves patient safety and quality of service. It reduces time and resources spent on data format conversion[3]

\section{References:}

1) R. H. Dolin, L. Alschuler, S. Boyer, C. Beebe, F. M. Behlen, P. V. Biron, and A. Shabo, "The HL7 Clinical Document Architecture," J. Am. Med. Inform. Assoc., vol. 13, no. 1, pp. 30-39, 2006.

2) R. H. Dolin, L. Alschuler, C. Beebe, P. V. Biron, S. L. Boyer, D. Essin, E. Kimber, T. Lincoln, and J. E. Mattison, "The HL7 Clinical Document Architecture," J. Am. Med. Inform. Assoc., vol, 8, pp. 552-569, 2001

3) M. L. M€uller, F. Ückert, and T. B€urkle, "Crossinstitutional data exchange using the clinical document architecture (CDA)," Int. J. Med. Inform., vol. 74, pp. 245-256, 2005.

4) H. Yong, G. Jinqiu, and Y. Ohta, "A prototype model using clinical document architecture (cda) with a japanese local "standard: designing and implementing a referral letter system," Acta Med Okayama, vol. 62, pp. 15-20, 2008 ..

5) K. Huang, S. Hsieh, Y. Chang, F. Lai, S. Hsieh, and H. Lee,"Application of portable cda for secure clinical-document exchange," J. Med. Syst., vol. 34, no. 4, pp. 531-539, 2010

6) C. Mart_nez-Costa, M. Men_arguez-Tortosa, and J. Tom_as Fern_andez- Breis, "An approach for the semantic interoperability of ISO EN 13606 and OpenEHR archetypes," J. Biomed. Inform., vol. 43, no. 5, pp. 736-746, Oct. 2010.

7) MR. Santos, MP. Bax, and D. Kalra, "Building a logical HER architecture based on ISO 13606 standard and semantic web technologies," Studies Health Technol. Informat., vol. 160, pp. 161- 165, 2010

8) K. Ashish, D. Doolan, D. Grandt, T. Scott, and D. W. Bates, "The use of health information technology in seven nations,'Int. J. Med. Informat., vol. 77, no. 12, pp. 848-854, 2008

9) G. J. Kuperman, J. S. Blair, R. A. Franck, S. Devaraj, and A. F. Low, "Developing data content specifications for the nationwide health information network trial implementations," J. Am. Med. Inform.

10) C. Mart_nez-Costa, M. Men_arguez-Tortosa, and J. Tom_as Fern_andez- Breis, "An approach for the semantic interoperability of ISO EN 13606 and OpenEHR archetypes," J. Biomed. Inform., vol. 43, no. 5, pp. 736-746, Oct. 2010.

11) MR. Santos, MP. Bax, and D. Kalra, "Building a logical HER architecture based on ISO 13606 standard and semantic web technologies," Studies Health Technol. Informat., vol. 160, pp. 161- 165, 2010.

12) K. Ashish, D. Doolan, D. Grandt, T. Scott, and D. W. Bates, "The use of health information technology in seven nations,"'Int. J. Med. Informat., vol. 77, no. 12, pp. 848-854, 2008.

13) G. J. Kuperman, J. S. Blair, R. A. Franck, S. Devaraj, and A. F. Low, "Developing data content specifications for the nationwide health information network trial implementations," J. Am. Med. Inform.

14) www.wikipedia.org/history.

15) http://en.wikipedia.org/wiki 